\title{
Prognostic value of left ventricular diastolic function and association with heart rate variability after a first acute myocardial infarction
}

\author{
S H Poulsen, S E Jensen, J E Møller, K Egstrup
}

\begin{abstract}
Objective-To study the prognostic value of left ventricular (LV) diastolic function and its relation with autonomic balance expressed by heart rate variability (HRV) in patients after a first acute myocardial infarction.

Design-The study population consisted of 64 consecutive patients with first acute myocardial infarction and 31 control subjects. Long and short term HRV indices were evaluated by 24 hour Holter monitoring, and LV systolic and diastolic function were assessed by two dimensional and Doppler echocardiography before discharge. Patients were divided into two groups: those with restrictive LV filling characteristics (deceleration time $\leqslant 140 \mathrm{~ms}$ ) and those with non-restrictive LV filling characteristics (deceleration time $>140 \mathrm{~ms}$ ).

Results-Both long and short term HRV indices were significantly reduced in patients with restrictive LV filling compared with the non-restrictive group and control subjects. Mitral deceleration time and isovolumetric relaxation time correlated weakly but significantly with all indices of HRV whereas ejection fraction correlated weakly with the long term HRV indices. The mean follow up time was 14.9 (8.7) months. Multivariate analysis showed that mitral deceleration time $\left(\chi^{2}=6.4, p<0.001\right)$ and ejection fraction $\leqslant 40 \%\left(\chi^{2}=4.4, p<0.05\right)$ were independent predictors of cardiac death and readmission to hospital with congestive heart failure.

Conclusions-A restrictive LV filling pattern was found to be the strongest predictor of adverse outcome independent of HRV and ejection fraction during follow up after a first acute myocardial infarction. Patients with restrictive LV filling characteristics had more reduced HRV than those with non-restrictive diastolic filling.

(Heart 2001;86:376-380)
\end{abstract}

Keywords: diastole; infarction; autonomic balance

Left ventricular (LV) systolic function is a well established predictor of morbidity and mortality following acute myocardial infarction. ${ }^{12}$ In recent years, $\mathrm{LV}$ diastolic dysfunction has been related to development of heart failure, progressive LV dilatation, and mortality after myocardial infarction..$^{3-7}$ Pulsed Doppler echocardiography has become well accepted as a reliable and useful non-invasive method for assessment of LV diastolic function. ${ }^{8}$ Mitral and pulmonary venous flow velocities assessed by Doppler are used for the evaluation of LV filling pressure, relaxation, and chamber stiffness in establishing diagnosis and prognosis. ${ }^{89}$ The identification of a restrictive LV filling pattern characterised by increased early to late filling ratio, decreased mitral deceleration time, and decreased systolic pulmonary venous flow has been associated with poor outcome in cardiac disease. ${ }^{89}$

Reduced heart rate variability (HRV) after myocardial infarction is an important risk factor for mortality and life threatening arrhythmias. ${ }^{10-13}$ Changes in HRV are believed to reflect an imbalance between sympathetic and parasympathetic activity. ${ }^{14}{ }^{15}$ Increasing LV wall stress by raising loading conditions may enhance LV dilatation and increase sympathetic activity leading to the process of LV remodelling. ${ }^{16}{ }^{17}$ In previous studies HRV was shown to be related to $\mathrm{LV}$ systolic function expressed by ejection fraction. ${ }^{9}{ }^{10}$ Furthermore, HRV has been related to in hospital heart failure and with progressive LV dilatation after anterior myocardial infarction. ${ }^{9} 101819$ In this study, we investigated the prognostic implications LV diastolic function assessed by Doppler echocardiography and its relation to HRV before discharge after a first myocardial infarction.

\section{Methods}

PATIENTS

We prospectively studied 64 consecutive patients admitted to the coronary care unit with first acute myocardial infarction defined as follows: (1) creatine kinase $\geqslant 210$ IU and creatine kinase fraction $B \geqslant 20$ IU (normal upper limit $=6 \mathrm{U} / \mathrm{l})$; (2) typical chest pain; and (3) electrocardiographic evidence of myocardial infarction. Another 31 patients admitted with suspected acute myocardial infarction, which was disproved by lack of enzyme release and electrocardiographic changes, served as a control group. These patients had no prior history of myocardial infarction but were considered to have angina pectoris as they had at least one of the following characteristics besides typical chest pain: (a) positive exercise test; (b) coronary angiogram with at least one stenotic lesion exceeding $75 \%$; or (c) previous treatment with percutaneous transluminal coronary angioplasty. Eligible for the study were patients in sinus rhythm, aged 40-75 years, and without valve disease. Patients continued their usual medication with the exception of $\beta$ blocker 
treatment, which was started after the Holter monitoring and echocardiography were performed. No patients received antiarrhythmic agents. All patients were planned for at least one year of follow up. The local scientific ethical committee approved the study and each participant gave informed written consent.

ASSESSMENT OF HRV

HRV was assessed at day $5-7$ by a 24 hour tape recording using a Tracker TR1 Holter monitor (Reynolds Medical, Herts, UK). The tape recordings were analysed for HRV by a Reynolds pathfinder 600 (version 4.12, Reynolds Medical Ltd). The tapes were manually edited to ensure deletion of noise and correct identification and classification of the QRS complexes before the analysis was performed. The following indices of HRV were assessed according to current recommendations: SD of all normal to normal (NN) intervals, the HRV triangular index, $\mathrm{SD}$ of the averages of $\mathrm{NN}$ intervals in five minute segments, the square root of the mean of the sum of the squares of differences between adjacent NN intervals, and coefficients of variance (SD of $\mathrm{NN}$ intervals divided by mean heart rate).$^{20}$

\section{ECHOCARDIOGRAPHY}

Two dimensional and pulsed Doppler echocardiographic examinations were performed at days 5-7 using a standard cardiac ultrasound unit with a $2.5 \mathrm{MHz}$ transducer. LV end systolic and diastolic volumes and ejection fraction were measured from the apical two and four chamber views by Simpson's biplane disc method. The mean of three measurements was used and volumes were indexed for body surface area. Pulsed Doppler recordings of the mitral flow velocities were obtained from the

Table 1 Baseline characteristics of control subjects and patients with or without restrictive left ventricular diastolic filling patterns

\begin{tabular}{lccr}
\hline & Control & Non-restrictive & Restrictive \\
\hline Number & 31 & 47 & 17 \\
Age (years) & $62(8)$ & $61(8)$ & $60(11)$ \\
Men (\%) & $18(58)$ & $34(72)$ & $13(76)$ \\
Diabetes mellitus (\%) & $2(6)$ & $3(6)$ & $1(6)$ \\
Hypertension (\%) & $9(29)$ & $10(21)$ & $4(24)$ \\
Q wave infarction (\%) & & $27(57)$ & $11(64)$ \\
Anterior infarction (\%) & & $17(36)$ & $7(41)$ \\
Creatine kinase B fraction (U/l) & & $103(102)$ & $121(91)$ \\
Thrombolytic treatment (\%) & & $28(60)$ & $12(70)$
\end{tabular}

Table 2 Echocardiographic data

\begin{tabular}{lccc}
\hline & Control & Non-restrictive & Restrictive \\
\hline E:A ratio & $0.93(0.29)$ & $0.92(0.27)$ & $1.56(0.32)^{\star \star}$ \\
DT (ms) & $188(41)$ & $196(44)$ & $117(13)^{\star \star}$ \\
IRT (ms) & $82(20)$ & $89(18)$ & $62(12)^{\star \star}$ \\
S (cm/s) & $52(7)$ & $46(9)$ & $39(11)^{\star \star}$ \\
D (cm/s) & $38(10)$ & $37(9)$ & $48(10)^{\star \star}$ \\
R (cm/s) & $21(3)$ & $25(3)$ & $30(4)^{\star \star}$ \\
AD-RD $(\mathrm{ms})$ & $29(20)$ & $26(18)$ & $-15(10)^{\star \star}$ \\
EDVI (ml/m & $62(21)$ & $73(18) \dagger$ & $93(17)^{\star \star}$ \\
ESVI $\left(\mathrm{ml} / \mathrm{m}^{2}\right)$ & $29(11)$ & $37(13) \dagger \dagger$ & $43(10)^{\star \star}$ \\
EF $(\%)$ & $61(9)$ & $51(8) \dagger \dagger$ & $43(10)^{\star \star}$ \\
\hline
\end{tabular}

Values are expressed as mean (SD).

${ }^{\star} \mathrm{p}<0.05 ;{ }^{\star \star} \mathrm{p}<0.01$ restrictive versus non-restrictive; $\mathrm{tp}<0.05,+\dagger \mathrm{p}<0.01$ non-restrictive versus control.

$\mathrm{AD}-\mathrm{RD}$, mitral A wave duration minus pulmonary venous flow reversal duration; $\mathrm{D}$, diastolic pulmonary peak flow velocity; DT, mitral E deceleration time; E:A ratio, early to late peak filling velocity; EDVI, end diastolic volume indexed for body surface; EF, left ventricular ejection fraction; ESVI, end systolic volume indexed for body surface; IRT, isovolumetric relaxation time; R, pulmonary venous flow reversal velocity; $S$, systolic pulmonary venous flow velocity. apical four chamber view by placing the sample volume between the tips of the mitral leaflets and pulmonary venous flow velocities were obtained by placing the sample volume at least $1 \mathrm{~cm}$ into the right superior pulmonary vein. Each Doppler profile was analysed by digital tracing and Doppler measurements were calculated from an average of five consecutive cardiac cycles. The pulmonary flow tracings were recorded in $83 \%$ of all cases. On the basis of previous combined invasive and echocardiographic studies in patients with coronary artery disease, the patients with acute myocardial infarction were assigned to two groups according to the mitral deceleration time. ${ }^{8921-23}$ Patients with mitral deceleration time $>140 \mathrm{~ms}$ were considered to have non-restrictive LV filling and patients with a deceleration time $\leqslant 140 \mathrm{~ms}$ to have restrictive LV filling. All echocardiographic and HRV measurements were analysed without knowledge of the clinical data.

STATISTICAL ANALYSIS

Results are presented as mean (1SD). Differences between groups were examined by Student's $t$ test for unpaired data. Correlations were assessed by bivariate correlation analysis. Univariate and multivariate regression analyses were applied to identify predictors of the combined end point of cardiac death or readmission to hospital with heart failure among baseline characteristics, HRV, and LV function. Kaplan Meier curves were constructed and compared by $\log$ rank tests. Values of $p<0.05$ were considered significant.

\section{Results}

Table 1 describes the clinical characteristics of the control group and patients with acute myocardial infarction, with or without LV restrictive filling characteristics. No significant differences were observed between groups except that there were more men in the group with myocardial infarction than in the control group $(p=0.05)$. Table 2 shows the echocardiographic data for each group. As expected the Doppler parameters of diastolic function differed between the restrictive group and the non-restrictive and control groups. However, no differences in Doppler data were noted between the non-restrictive and control groups. LV ejection fraction was significantly lower among patients with acute myocardial infarction than in controls. In the restrictive group LV ejection fraction was $\leqslant 40 \%$ in 10 patients whereas seven patients had an ejection fraction $>40 \%$. In the non-restrictive group 40 patients had an ejection fraction $>40 \%$ and seven patients had an ejection fraction $\leqslant 40 \%$.

The systolic blood pressure was 128 (22) $\mathrm{mm} \mathrm{Hg}$ in the control group, 131 (21) $\mathrm{mm} \mathrm{Hg}$ in the non-restrictive group, and 126 (19) $\mathrm{mm} \mathrm{Hg}$ in the restrictive group (NS).

ASSOCIATION BETWEEN HRV INDICES AND LV FUNCTION

The results of the Holter examination of HRV between groups are listed in table 3 .

A weakly significant correlation was found between the SD of the $\mathrm{NN}$ intervals and 
Table 3 Heart rate variability $(H R V)$ data

\begin{tabular}{lclc}
\hline & Control & Non-restrictive & Restrictive \\
\hline Mean heart rate (beats/min) & $72(10)$ & $73(10)$ & $77(10)^{\star}$ \\
HRV index & $32(10)$ & $28(9)$ & $22(8)^{\star \star}$ \\
SDNN (ms) & $114(40)$ & $99(21)$ & $70(13)^{\star \star}$ \\
SDANN (ms) & $89(26)$ & $85(25)$ & $71(25)^{\star \star}$ \\
RMSSD (ms) & $30(14)$ & $27(13)$ & $23(11)^{\star \star}$ \\
CV (\%) & $14(5)$ & $12(3)$ & $9(2)^{\star \star}$
\end{tabular}

Values are expressed as mean (SD).

${ }^{\star} \mathrm{p}<0.05,{ }^{\star \star} \mathrm{p}<0.01$ restrictive versus non-restrictive.

$\mathrm{CV}$, coefficients of variance $=\mathrm{SD}$ of the $\mathrm{NN}$ intervals $(\mathrm{SDNN}) /$ mean heart rate; HRV index, HRV triangular index; RMSSD, the square root of the mean of the sum of the squares of differences between adjacent $\mathrm{NN}$ intervals; SDANN, SD of the averages of $\mathrm{NN}$ intervals in 5 minute segments.

isovolumetric relaxation time $(r=0.25$, $\mathrm{p}=0.05)$, peak systolic pulmonary venous flow $(r=0.30, \mathrm{p}=0.01)$, and ejection fraction $(r=0.24, \mathrm{p}=0.05)$ whereas no correlation was found with early to late peak filling velocity or LV volume (table 4). Figure 1 presents the association between mitral deceleration time and the SD of the NN intervals. The long term HRV expressed by the SD of the averages of $\mathrm{NN}$ intervals in five minute segments correlated significantly with mitral deceleration time $(r=0.31, \mathrm{p}=0.01)$, isovolumetric relaxation time $(r=0.25, \mathrm{p}=0.05)$, and ejection fraction $(r=0.24, \mathrm{p}=0.05)$. The SD of the averages of NN intervals divided by mean heart rate correlated significantly with mitral deceleration time $(r=0.26, \mathrm{p}<0.05)$ and isovolumetric relaxation time $(r=0.24, \mathrm{p}<0.05)$ but not ejection fraction $(r=0.13, \mathrm{NS})$. The short term variability expressed by the square root of the mean of the sum of the squares of differences between adjacent NN intervals was significantly correlated with mitral deceleration time $(\mathrm{r}=0.23, \mathrm{p}=0.05)$, isovolumetric relaxation time $(r=0.27, \mathrm{p}=0.01)$, and ejection fraction $(r=0.26, \mathrm{p}=0.05)$.

PREDICTIVE CHARACTERISTICS OF LV SYSTOLIC AND DIASTOLIC FUNCTION AND HRV DURING FOLLOW UP

The mean follow up time was 14.9 (8.7) months during which nine cardiac deaths (five sudden deaths, four from progressive heart failure) occurred and eight patients were readmitted to hospital with congestive heart failure. Univariate analyses showed that mitral deceleration time $\leqslant 140 \mathrm{~ms}\left(\chi^{2}=8.5, \mathrm{p}<0.001\right)$, $\mathrm{LV}$ ejection fraction $\leqslant 40 \% \quad\left(\chi^{2}=6.2\right.$, $\mathrm{p}=0.001), \mathrm{SD}$ of the averaged $\mathrm{NN}$ intervals

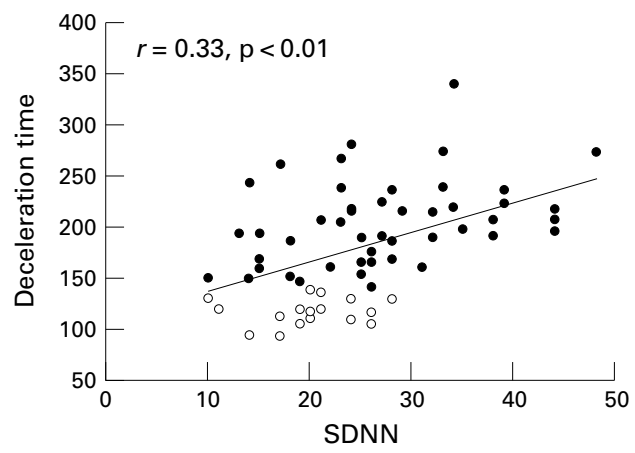

Figure 1 Association between mitral deceleration time and $S D$ of normal to normal (NN) intervals. Open circles denote patients with non-restrictive left ventricular filling and closed circles denote patients with restrictive filling.
Table 4 Relation between left ventricular systolic and diastolic function and HRV (standard deviation of NN intervals)

\begin{tabular}{lcl}
\hline & Correlation coefficient & $p$ Value \\
\hline E:A ratio & 0.03 & NS \\
DT & 0.33 & 0.001 \\
IRT & 0.25 & 0.05 \\
S & 0.30 & 0.01 \\
D & 0.10 & NS \\
R & -0.29 & 0.05 \\
EDVI & 0.08 & NS \\
ESVI & 0.11 & NS \\
EF & 0.24 & 0.05 \\
\hline
\end{tabular}

See table 2 for key to abbreviations.

$\leqslant 92\left(\chi^{2}=4.4, \mathrm{p}<0.01\right)$, peak creatine kinase $\mathrm{B}$ fraction $\left(\chi^{2}=3.3 \mathrm{p}=0.01\right)$, and age $\left(\chi^{2}==1.07 /\right.$ year increase, $\left.p<0.02\right)$ were significant predictors of cardiac events during follow up. Multivariate analysis showed that mitral deceleration time $\left(\chi^{2}=6.4, \mathrm{p}<0.001\right)$ and ejection fraction $\leqslant 40 \% \quad\left(\chi^{2}=4.4\right.$, $\mathrm{p}<0.05)$ were independent predictors of outcome.

Figure 2 shows the event free survival curves for mitral deceleration time, ejection fraction, and SD of NN intervals.
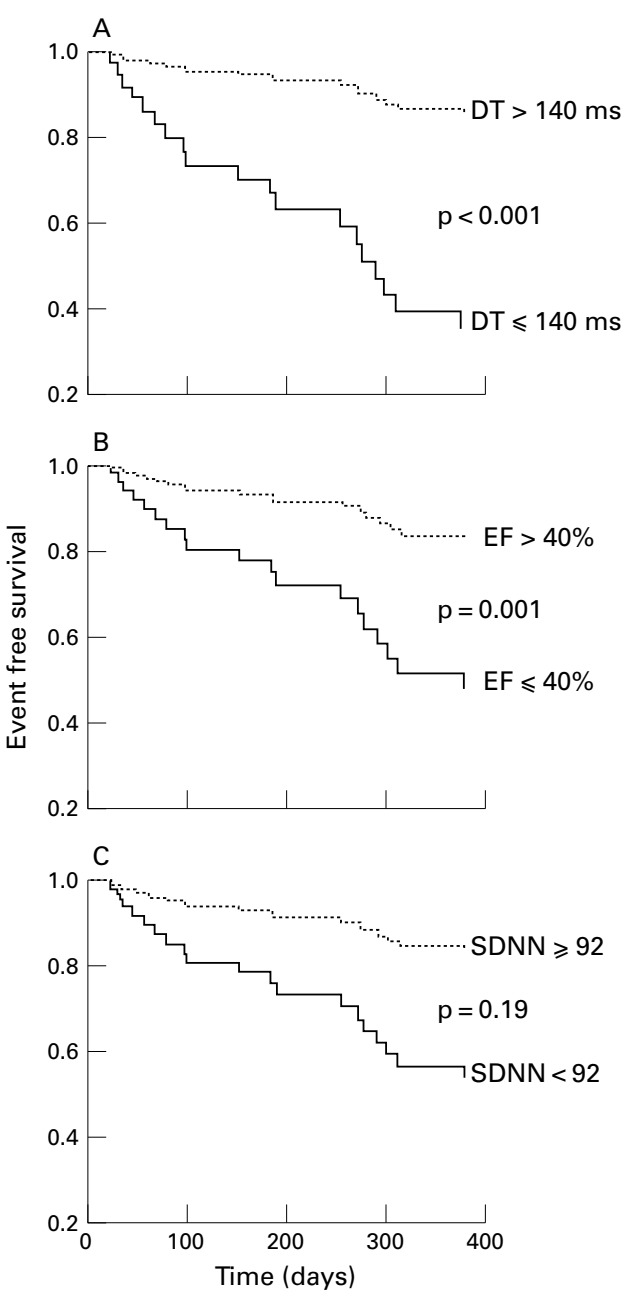

Figure 2 Cumulative event free survival from cardiac death or readmission to hospital with congestive heart failure in (A) patients with acute myocardial infarction with mitral deceleration time $(D T) \leqslant 140 \mathrm{~ms}$ and $>140 \mathrm{~ms}$,

(B) patients with ejection fraction $(E F) \leqslant 40 \%$ and $>40 \%$, and $(C)$ patients with SD of all NN intervals $\geqslant 92$ and $<92$. 


\section{Discussion}

In the present study a shortened mitral deceleration, representing a restrictive $\mathrm{LV}$ filling pattern compared with HRV and ejection fraction, was found to be the best predictor of outcome following a first myocardial infarction. Patients with restrictive LV filling have significantly reduced HRV compared with patients with a non-restrictive LV filling pattern and control subjects. Total, long term, and short term HRV indices correlated weakly but significantly with parameters of LV diastolic function.

Diastolic function of the heart is a complex sequence of many interrelated determinants where the two major factors of LV filling are ventricular relaxation and the effective chamber compliance. Both factors are known to be influenced by myocardial ischaemia, hypertrophy, fibrosis, increased afterload, and regional asynchrony. ${ }^{8}$ Doppler echocardiography has identified three abnormal LV filling patterns: impaired relaxation, and pseudonormal and restrictive filling patterns. Impaired LV relaxation is seen early, is associated with acute myocardial ischaemia, LV hypertrophy, and increasing age, and is recognised by a decrease in early to late peak filling velocity, and prolonged deceleration and isovolumetric relaxation times. As the diastolic abnormalities progress, increased LV chamber stiffness and atrial pressure lead to a pseudonormal filling pattern that can be unmasked by careful evaluation of pulmonary venous flow or the use of the Valsalva manoeuvre. When pronounced diastolic abnormalities are present, a restrictive filling pattern develops characterised by increased early to late peak filling velocity, and shortened deceleration and isovolumetric relaxation times due to increased left atrial pressure and increased LV filling pressure. Patients with restrictive LV filling often have reduced functional capacity and are associated with a poor prognosis. ${ }^{8}$ In the present study we identified a restrictive filling pattern in approximately one fifth of the patients, which is in accordance with previous findings. ${ }^{7}$ Restrictive LV filling is often seen in patients with moderate to severe systolic dysfunction, which is consistent with our findings. However, in the present study a minor group with restrictive LV filling had preserved or only mildly affected systolic dysfunction indicating that diastolic dysfunction might exist independent of moderate to severe systolic dysfunction after myocardial infarction.

The clinical implications of restrictive LV filling with preserved systolic function cannot be determined by the present study, because of the size of the study population, but must clarified by future studies. According to the diastolic pressure-volume curve, a decrease in effective chamber compliance can be caused by either increased myocardial stiffness (or increased pericardial restraint) or an increase in volume. In the present study significant early LV dilatation was seen in patients with restrictive filling, which is likely to be an important determinant in the development of increased chamber compliance. Myocardial necrosis, interstitial oedema, and increased collagen deposition might also affect myocardial stiffness. ${ }^{24}$ Myocardial ischaemia might also be indirectly involved as patency of the infarct related artery is an important factor in avoiding progressive LV dilatation. ${ }^{25}$ During acute myocardial ischaemia an impaired relaxation filling pattern is usually seen, which might be involved in the early stage of the development of diastolic dysfunction. ${ }^{26}$ The development of a restrictive LV filling pattern and early $\mathrm{LV}$ dilatation is likely to increase myocardial wall stress, which might lead to progressive LV dilatation and sympathetic activation. The presence of both a shortened deceleration time and reduced HRV have previously been related to LV dilatation after myocardial infarction..$^{5619}$ In the present study the total and long term HRV indices were significantly altered in patients with restrictive LV filling compared with patients with non-restrictive LV filling or control subjects. Our observations are supported by experimental studies in dogs, which showed that impairment of LV diastolic function is followed by a parallel deterioration of autonomic regulation. ${ }^{27}$ In the clinical setting reduced HRV has been found to be associated with abnormal myocardial performance as it is significantly correlated with decreased LV ejection fraction and development of congestive heart failure following myocardial infarction. ${ }^{1011}$

HRV has previously been associated with late diastolic filling during atrial contraction but appeared to be independent of measures of early diastolic filling in normal subjects. ${ }^{28}$ In the present study we did not find any significant correlation between HRV and early or late peak filling velocity but we did find a significant correlation with deceleration time, isovolumetric relaxation time, and peak systolic pulmonary venous flow velocity. This discrepancy might be caused by altered loading conditions, which is supported by studies in normal subjects in whom both early and late peak filling velocities are found to be more affected than other diastolic parameters by changes in preload. ${ }^{29}$ In accordance with previous studies we found a weak but significant correlation between LV ejection fraction and total HRV indices but not with short term HRV. ${ }^{13}$

\section{PREDICTIVE CHARACTERISTICS OF EJECTION} FRACTION, DECELERATION TIME, AND HRV DURING FOLLOW UP

Reduced HRV and ejection fraction have been shown to be independent predictors of all cause mortality after myocardial infarction, even though HRV appears to be a better predictor of postinfarction arrhythmic complications including sudden death. In the present study, we found that deceleration time $(\leqslant 140 \mathrm{~ms})$ and ejection fraction were predictors of a combined end point of cardiac death and readmission to hospital with heart failure during follow up. In contrast to previous studies reduced HRV was not found to be an independent predictor of outcome. No measures of LV diastolic function are available from earlier studies or included in their analysis, and total cardiac mortality was used as the end point in 
a considerably larger number of patients. This might explain the discrepancy in the predictive value of measures of HRV and LV systolic and diastolic function between the present and previous studies.

\section{LIMITATIONS}

The correlation analysis between diastolic Doppler parameters and HRV indices has some inherit problems as each parameter does not describe solely the different stages of diastolic function or autonomic balance, which is an important limitation. However, the HRV indices were significantly different between groups and it is therefore likely that some interaction is present. Diastolic Doppler parameters and HRV are both influenced by age and heart rate, which must be taken into consideration in the interpretation of the present results. ${ }^{30}{ }^{31}$ We do believe that the influence of heart rate is limited as only patients between 40 and 75 years of age were included in the present study. Patients with shortened deceleration time ( $\leqslant 140 \mathrm{~ms}$ ) had significantly higher heart rate than patients with nonrestrictive filling characteristics. Increasing heart rate has less influence on deceleration time and at most prolongs the deceleration time, which limits the possibility of misclassification of patients with restrictive filling pattern into the non-restrictive LV filling group. HRV determined by the SD of averages of NN intervals corrected for mean heart rate was also significantly different between those patients with and those without restrictive filling characteristics. Furthermore, all patients were studied without ongoing $\beta$ blocker treatment, which is known to influence heart rate, HRV, and diastolic LV filling. ${ }^{32}{ }^{33}$ Angiography and invasive revascularisation procedures were not performed routinely in this study and so vessel patency was unknown.

\section{CONCLUSION}

The presence of a restrictive LV filling pattern was found to be the strongest predictor of adverse outcome independent of HRV and ejection fraction during follow up after a first acute myocardial infarction. Patients with restrictive LV filling characteristics were shown to have more reduced HRV than those with non-restrictive diastolic filling and control subjects.

1 Pfeffer MA, Braunwald E, Moyé LA, et al. Effect of captopril on mortality and morbidity in patients with LV dysfunction after acute myocardial infarction. Results of the Survival and Ventricular Enlargement Trial. $N$ Engl $f \mathrm{Med}$ 1992;327:669-77.

2 Volpi A, De Vita C, Franzosi MG, et al. Determinants of 6-month mortality in survivors of myocardial infarction 6-monthis after thrombolysis. Resi

3 Oh JK, Ding ZP, Gersh BJ, et al. Restrictive LV diastolic filling identifies patients with heart failure after acute myocaring identifies patients with heart failure after acute myo

4 Poulsen SH, Jensen SE, Gøtzsche O, et al. Evaluation and prognostic significance of LV diastolic function assessed by prognostic significance of LV diastolic function assessed by Doppler echocardiography in the very early phase of

5 Cerisano G, Bolognese L, Carrabba N, et al. Doppler derived mitral deceleration time. An early strong predicto of LV remodelling after reperfused anterior acute myocardial infarction. Circulation 1999;99:230-6.
6 Poulsen SH, Jensen SE, Egstrup K. Longitudinal changes and prognostic implications of LV diastolic function in first acute myocardial infarction. Am Heart f 1999;137:910-8.

7 Nijland F, Kamp O, Karreman AJP, et al. Prognostic implications of restrictive LV filling in acute myocardial infarction: a serial Doppler echocardiographic study. $\mathcal{F ~} \mathrm{Am}$ Coll Cardiol 1997;30:1618-24.

8 Nishimura RA, Tajik AJ. Evaluation of diastolic filling of left ventricle in health and disease: Doppler echocardiography is the clinician's Rosseta stone. F Am Coll Cardiol 1997;30: $8-18$.

9 Appleton CP, Hatle LK. The natural history of LV filling abnormalities: assessed by two-dimensional and Doppler abnormalities: assessed by two-dimensional and Dopp

10 Kleiger RE, Miller JP, Bigger JT, et al. Decreased HRV and its association with increased mortality after acute myocardial infarction. Am f Cardiol 1987;59:256-62.

11 Odemuyiwa O, Malik M, Farrell T, et al. Comparison of the predictive characteristics of HRV index and LV ejection fraction for all-cause mortality, arrhythmic events and sudden death after acute myocardial infarction. Am $\mathcal{F}$ Cardiol 1991;68:434-9

12 Farrall TG, Bashir Y, Cripps T, et al. Risk stratfication for arrhythmic events in postinfarction patients based on HRV, ambulatory electrocardiographic variables and signalaveraged electrocardiogram. F Am Coll Cardiol 1991;18: 687-97.

13 Vaishnav S, Stevenson R, Marchant B, et al. Relation between HRV early after myocardial infarction and long-term mortality. Am f Cardiol 1994;73:653-7.

14 Ewing DJ. Cardiovascular reflexes and autonomic neuropathy. Clin Sci Mol Med 1978;55:321-7.

15 Katona PG, Jih F. Respiratory sinus arrhythmia:noninvasive measure of parasympathetic cardiac control. F Appl Physiol 1975;39:801-5

16 Pierard LA, Albert A, Gilis F, et al. Hemodynamic profile of patients with acute myocardial infarction at risk of infarct expansion. Am f Cardiol 1987;60:5-9.

17 Pfeffer MA, Braunwald E. Ventricular remodelling after myocardial infarction. Experimental observations and clinical implications. Circulation 1990; 81:1161-72.

18 Odemuyiwa O, Poloniecki J, Malik M, et al. Temporal influences on the prediction of postinfarction mortality by HRV: a comparison with LV ejection fraction. Br Heart 7 1994;71:521-7.

19 Dambrink J-HE, Tuininga YS, van Gilst WH, et al. Association between reduced HRV and LV dilatation in patients with a first myocardial infarction. Br Heart $\mathcal{F} 1994 ; 72$ :51420.

20 Task Force of the European Society of Cardiology and the North American Society of Pacing and Electrophysiology. HRV. Standards of measurement, physiological interpretation, and clinical use. Circulation 1996;93:1043-65.

21 Cohen GI, Pietrolungo JF, Thomas JD, et al. A practical guide to assessment of ventricular diastolic function using Doppler echocardiography. F Am Coll Cardiol 1996;27: 1753-60.

22 Cecconi M, Manfrin M, Zanoli R, et al. Doppler echocardiograhic evaluation of LV end-diastolic pressure in patients with coronary artery diasease. $\mathcal{F}$ Am Soc Echocardiogr 1996; 24:241-50.

23 Rakowski H, Appleton C, Chan KW, et al. Canadian consensus recommendations for the measurements and reporting of diastolic dysfunction by echocardiography. $\mathcal{F}$ Am Soc Echocardiogr 1996;9:736-60.

24 Poulsen SH, Høst NB, Jensen SE, et al. Relationship between serum amino-terminal propeptide of type III probetween serum amino-terminal propeptide of type III procollagen and changes of left ventricular function after

25 Popovic AD, Neskovic AN, Babic R, et al. Independent impact of thrombolytic therapy and vessel patency on LV dilation after myocardial infarction. Circulation 1994;90: $800-7$.

26 DeBruyne B, Lerch R, Meier B, et al. Doppler assessment of eft ventricular filling during brief coronary occlusion. Am Heart $\mathcal{F}$ 1989;117:629-35.

27 Ishise $\mathrm{H}$, Asanoi $\mathrm{H}$, Ishizaka S, et al. Time course of sympathovagal imbalance and LV dysfunction in conscious dogs with heart failure. F Appl Physiol 1998;84:1234-41.

28 Finkelhor RS, Karwa P, Kaufman ES, et al. The relationship of autonomic function to age-related changes in LV filling of autonomic function to age-related changes in LV
in normal subjects. Eur Heart f 1995;16:1716-20.

29 Møller JE, Poulsen SH, Egstrup K. Effects of preload alternations on a new Doppler echocardiographic index of combined systolic and diastolic performance. $7 \mathrm{Am}$ Soc Echocardiogr 1999;135:1065-72.

30 Tsuji H, Venditti FJ, Manders ES, et al. Determinants of HRV. F Am Coll Cardiol 1996;28:1539-46.

31 Harrison MR, Clifton GD, Pennell AT, et al. Effect of heart rate on LV diastolic transmitral flow velocity patterns assessed by Doppler echocardiography in normal subjects. Am 7 Cardiol 1991;67:622-7.

32 Molgaard H, Mickley H, Pless P, et al. Effects of metoprolol on HRV in survivors of acute myocardial infarction. Am $\mathcal{F}$ on HRV in survivors of

33 Poulsen SH, Jensen SE, Egstrup K. Effects of long-term adrenergic $\beta$-blockade on LV diastolic filling in patients with acute myocardial infarction. Am Heart f 1999;138: $710-20$. 